\title{
Recent Developments on Shape Memory Applications
}

\author{
P.A. Besselink \\ Memory Metal Holland, Gronausestraat 1220, 7534 AT Enschede, The Netherlands
}

\begin{abstract}
In this paper some recently developed applications, using all kinds of possibilities that are offered by shape memory effects, will be presented.

The overview is meant to show the wide range of application fields, where a series of special properties of memory metals bring huge advantages and open new ways to develop products that normally could not be made with conventional, "non-living" materials.

During the last years the main issue in memory metal industries was the production of superelastic devices for medical use. However, the use in connectors, actuators and combined sensor/actuator functions in safety or control devices is also getting more important. In martensitic state memory metals are used for their extreme deformability compared to conventional metals. Temperature sensitive memory metal locking elements offer possibilities for the design of modular, adjustable devices like medical instruments and implants.
\end{abstract}

\section{INTRODUCTION}

A short summary of the properties of shape memory and superelastic alloys is given as an introduction to the applications that are presented in this article.

The behaviour of memory metals is caused by a change in the internal structure of the material by a martensitic transformation.

Extremely large deformations, applied at relatively low tempereture, can be neutralized in a small temperature range, because the material remembers its undeformed shape, in which it was programmed before by means of a proper heat treatment after cold working.

The driving energy for the shape memory effect is so high that a useful force is built up, if the recovery from the deformed state to the programmed shape is opposed.

In the next figures the most important properties are eludicated .

\subsection{Transformation temperatures}

These temperatures are merely determined by the chemical composition, but the fine tuning is achieved with the final thermomechanical treatment of the alloy.

This results in a typical structure-temperature relationship as shown in fig. 1, giving the reversible temperature induced transformation. Between the low-temperature martensitic structure and the high temperature austenitic structure a temperature hysteresis loop is shown, which is dependent on the alloy type.

During heating the transformation and thus the memory effect take place in the traject As-Af ( start and finish of the austenite formation respectively) and during cooling the reverse transformation to martensite occurs in the traject Ms-Mf.

Note that in the small temperature ranges As-Af and Ms-Mf the material has a mixed structure of martensite and austenite. Within the hysteresis loop the structure and shape of the product are dependent on the thermal history. It may be either martensitic and deformed if it comes from the lower temperature or austenitic and recovered if it comes from the high temperature side. 


\subsection{Superelasticity}

Above Af the material is completely austenitic and in that state it is only possible to induce the transformation to martensite by means of an externally applied stress. As soon as this external stress is removed, the reason for the transformation to stress induced martensite disappears and thus the material transforms back to the original austenitic structure. This always happens at a lower stress level, so there is a stress hysteresis between loading and unloading.

Fig. 2 shows a typical stress-strain curve of a superelastic NiTi-alloy, which can be deformed until $8 \%$ strain without permanent deformation. Loading and unloading curves both show a plateau, where the stress level is almost independent of the strain.

These values of the loading and unloading plateau vary with the testing temperature and are higher for increased temperature.

Superelasticity is only possible in a limited temperature range between Af and Md, as given in fig. 1. Generally $\mathrm{Md}$ is about $50 \mathrm{C}$ above Af. Above this point the critical stress for plastic deformation is reached below the upper plateau stress and therefore the material will be deformed permanently.

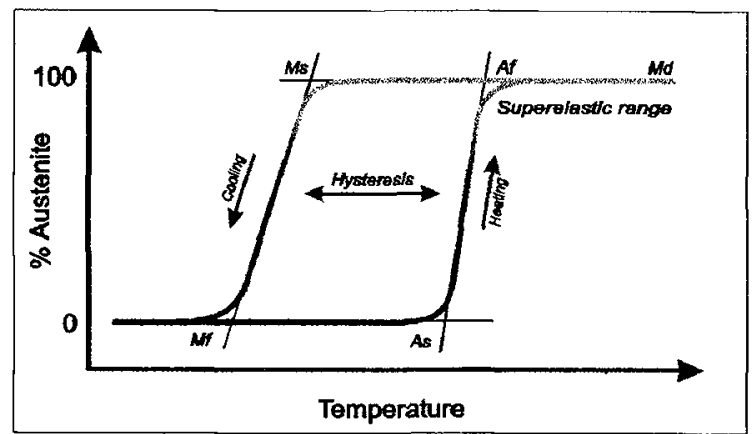

Figure 1: Influence of the temperature on the trans formation of martensite to austenite in a NiTi-shape memory alloy.

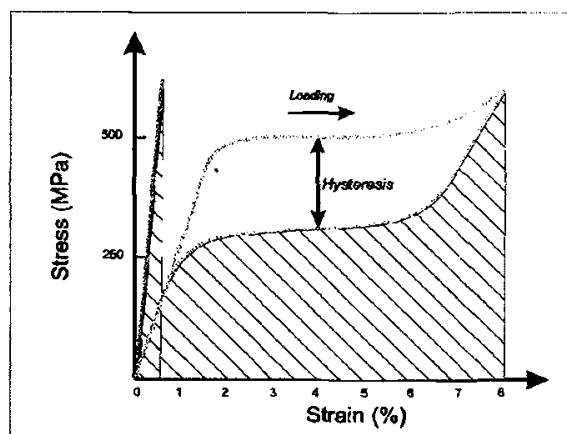

Figure 2: Stress-strain curve for steel and superelastic NiTi, where the dashed area's represent the stored energy for the same stress.

\subsection{Stored energy}

In fig. 2 a comparison is given between the capacity to store elastic energy for linear elastic material like steel and superelastic material. This elastic energy is represented as the dashed surface area between the curve and the strain axis. For steel this leads to a very small triangular surface area, compared to the much greater surface below the unloading curve of the superelastic material:

NiTi has a Young's modulus, which is a factor three lower than for steel and it can be loaded until a maximum stress level, which also is a factor two or three lower than for steel.

However, because of the enormous elastic recoverable strain, it can store 10 to 20 times more elastic energy.

\subsection{Recovery stress}

When the memory metal is deformed at a temperature below Mf and then released, it will stay in that configuration until it is heated above the transformation temperature range As-Af.

From As to Af the memory metal tries to return to the predefined shape, but if this free recovery is constrained, it will build up a recovery stress. In fig. 3 this recovery stress is shown as the vertical line which runs from the lower, unloaded and deformed position to the unloading plateau of the curve, which belongs to the higher temperature level. 
It may be clear that the resulting recovery stress is determined by the remaining strain and the final temperature.

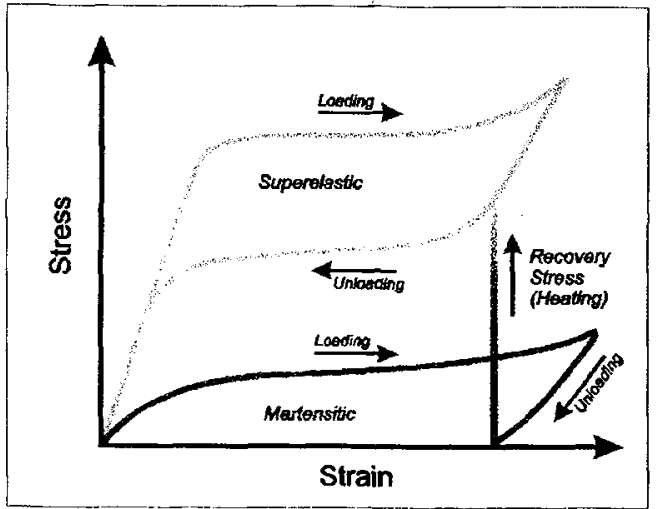

Figure 3: Stress strain curves for martensite and austenite. Building up of recovery stress when free recovery is constrained upon heating.

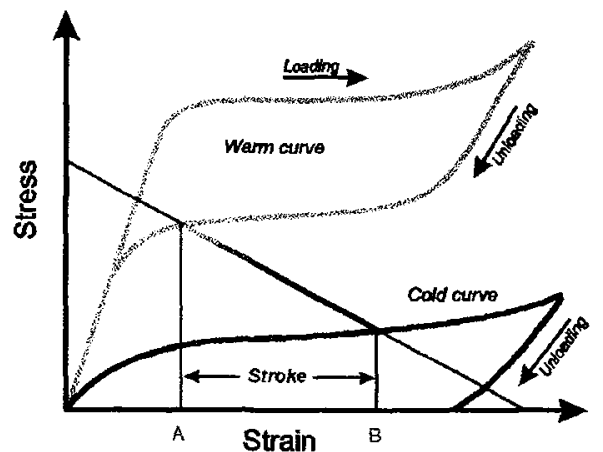

Figure 4: Characteristic of a NiTi-alloy working against a bias spring. Points A (warm) and B ( cold) define the available output stroke

\subsection{Working against a bias spring}

In many applications the transformation cycle has to be repeated several times and therefore it is desirable to use the memory metal in combination with a resetting element.

Fig. 4 gives an example of a warm, austenitic and a cold, martensitic curve, together with the characteristic of a linear elastic bias spring, which is mounted to the memory metal element.

If the memory metal is warm, it overcomes the biasing force of the spring and reaches strain A at the intersection point of the two characteristics. During cooling, the bias spring is strong enough to deform the memory metal until strain $B$ is reached.

Stroke A-B is the available output stroke, used to move an application between the warm (A) and cold (B) position.

\subsection{Deformation of martensitic material}

If the memory metal is deformed at a temperature below As, the deformation is caused by the formation of martensite variants that have a more favourable orientation to accommodate the applied stress. A deformation up to $8 \%$ strain does not lead to plastic deformation, but to a reorientation of the martensite. In principle this can be repeated many times without the excessive formation of dislocations like in conventional metals. This means that the fatigue resistance of memory metals in the range of large strains is much better.

\section{APPLICATIONS OF SENSOR/ACTUATOR FUNCTIONS}

Memory metals are often used for the combined function of a sensor and an actuator and offer a very compact drive system in control elements and safety devices. Sometimes it is possible to use a mechanical amplifier that enables the designer to use a restricted amount of memory metal that only works as a trigger to activate the system. This increases the reaction speed considerably.

Two examples are given of devices where a minimized amount of memory metal is used in such a way that is gives an optimal performance without a loss of functionality. 


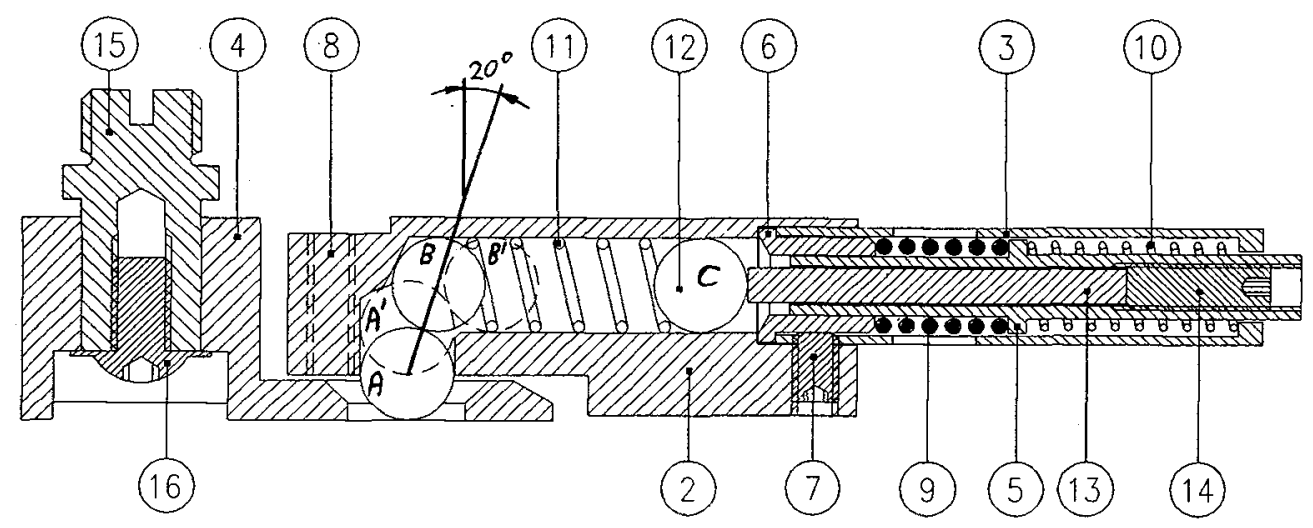

Figure 5: Fire door release mechanism with mechanical amplification of the forces of NiTi-(9) and bias (10) spring. When balls A and B move to positions A' and B', the slide block (4) can freely move to the left.

\subsection{Fire door release mechanism}

In many cases fire doors have to be held open during normal use, but in case of fire they have to close in order to prevent the fire entering from one room to another. The fire door works with a conventional door closer with a hydraulic damper and a slide block that moves through a track in the door frame. The system allows free movement of the door over an adjustable angle. At a specific position a hole in the slide block snaps under a set of spring loaded balls ( A and B in fig.5) in a so called thermolock and the door is held open. If desired the door can be closed again with a slight pressure until the ball snaps out of the slide block. The rest of the closing procedure is done by the door closer. Only in case of fire a sensor/actuator NiTi-spring 9 with an As-temperature of 40 degrees Celsius becomes active. It lifts control element 5 against the force of a steel preload spring 10 . The preload on the balls $A$ and $B$, caused by spring 11 disappears as soon as ball $\mathrm{C}$ moves to the right. Now the door closes automatically.

The set of spring loaded balls A and B, mounted under an angle of almost 70 degrees with the axis of movement of control element 5 , has the function of a mechanical amplifier for the force of the memory metal spring. Without this amplifier the NiTi-spring has to be 3 times stronger. This means that the system would be much heavier and so the reaction time in case of fire would have been much longer.

\subsection{Automatic adjustment of venetian blinds}

As seen in fig. 4 the stroke of an actuator, working against a bias spring can not be larger than stroke AB. The net force, available for steering the application is determined by the absolute difference between the actual load in the memory metal and in the bias spring.

In the ideal case the characteristic of the bias spring would have been almost horizontal, exactly between the loading characteristic of the martensitic material and the unloading characteristic of the austenitic material. Then the net available force for the application is optimal and the stroke can be larger. This is approached by the use of a constant-force spring that is combined with a memory metal sensor/actuator in the Solar Actuator, developed by IPS [ 1 ].

In fig. 6 a simple actuator is shown, made with a NiTi-torsion rod, mounted to a spring motor assembly with a torque that is invariant over an angle of 90 degrees. Around the NiTi-torsion rod a solar collector with a spectral selective layer is mounted, that heats the rod above ambient temperature as soon as direct sun light shines on the collector. The position of the venetian blinds is regulated automatically. 

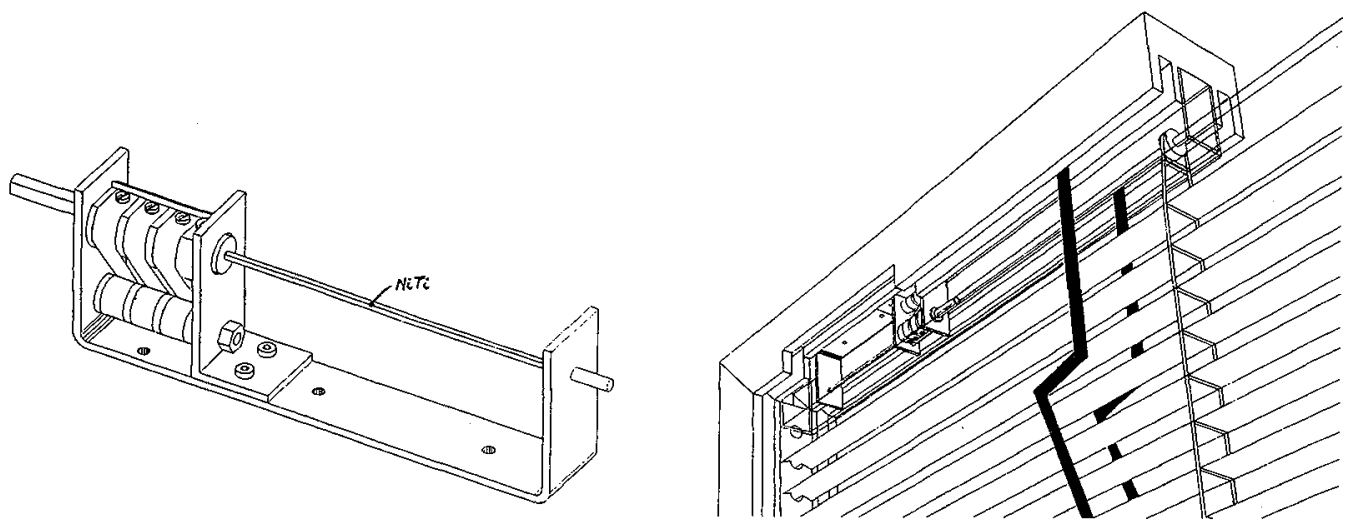

Figure 6: Use of a bias spring with an invariant force in combination with a NiTi-torsion rod in a Solar Actuator. The left figure gives the construction and the right figure shows the assembled unit in a window frame.

\subsection{Actuators}

$\mathrm{NiTi}$-actuators in the shape of wires, ribbons and springs are used in all kinds of systems and direct resistance heating is the most common way to control the position. The optimal geometry of such an actuator can be calculated when the needed force, stroke, amount of cycles, transformation temperatures and available power supply are known [ 2 ]. Examples of products are remote controlled robot systems, control elements for (micro-)valves, release mechanisms for space applications, bone distraction devices [ 3 ] and very small actuators in braille displays. By measuring the resistance during transformation, the actual position of the actuator can be defined and controlled [ 4 ].

The most common used principles for deformation in a NiTi-actuator are (combinations of) torsion, tension and bending. The most effective way to use the memory metal is where the material is loaded on a uniform stress. In case of bending or torsion only a small part of the material works at its optimum stress and strain level. However the advantage of larger displacement can be more important in some cases.

\subsubsection{Torsion}

A compact release mechanism for space applications uses a square NiTi-torsion rod of $2.5 \times 2.5 \mathrm{~mm}$ cross section, that turns a heavy bayonet joint over an angle of 45 degrees. After turning the bayonet is unlocked and the holding force of $2000 \mathrm{~N}$ is uncoupled.

\subsubsection{Tension}

Nepas developed a module for a braille display, using a standard miniature actuator with a straight NiTiCu wire with a length of $40 \mathrm{~mm}$ and a diameter of $0.17 \mathrm{~mm}$, used in tension. The size of one actuator, including the housing, is $2.2 \mathrm{~mm}$ diameter with a length of $50 \mathrm{~mm}$ and the stroke is $1 \mathrm{~mm}$ with a force of $1.5 \mathrm{~N}$. It needs 1.5 Volt and 0.4 Ampere. If these modules, with 8 actuators each, are built in a full page display, the amount of heat generated by thousands of actuators, causes problems.

Therefore the display uses a locking plate that fixates the position of the pins, once they have reached their final position. This enables the memory metal to cool down, while in the pins are in position. This increases the speed of the system and saves more than $90 \%$ of the required energy. 


\subsubsection{Bending}

Several types of miniature grippers are under development. Most types use a monolithic trained NiTielement with two way memory effect. An example of such a gripper is a remote controlled occluder for very small arteries, developed by Medalab. The memory element is an open ring with an external diameter of $3 \mathrm{~mm}$, embedded in silicon. The heating is achieved by indirect resistance heating and the free ends of the ring open $1 \mathrm{~mm}$ during cooling.

\section{CONNECTORS}

The use of NiTi in connectors is widespread. One of the first large applications was the Raychem connector sleeve for hydraulic tubing in airplanes. For this application the wide hysteresis alloy NiTiNb was developed. After transformation during heating, the recovery stress stays stable even at low temperatures. Later a series of connector rings for electrical and electronic devices came on the market. For medical applications, where the ambient temperature is more stable, there is less need for using these wide hysteresis alloys.

There are several ways to use memory metal elements in connectors:

1. An expanded ring or sleeve that contracts tightly on a rod or tube during heating.

2. A massive pin that expands during transformation and locks itself in a hole.

3. A clamp or gripper that opens or closes.

Some examples of different types of connectors are:

\subsection{Lens holder}

The leading end of a superelastic tube in an endoscope can hold an optical lens. After cooling below Mf the lens can be pressed into the end of the tube and after recovery the connection is very tight. With a proper surface of lens and tube this connection is leak tight and very insensitive to temperature variations during sterilisation and normal ambient temperature.

\subsection{Cylindrical connector pins}

A cylindrical, prestrained NiTi pin can be inserted into blind holes of the two opposing parts that have to be connected in an adjustable relationship. After heating up to body temperature the diameter of the contracting pin increases and the pin locks itself tightly into the holes. Axial compression is also occurring automatically. These connectors can be used for modular implants, like with threadless anchoring of abutments to dental root implants.

\subsection{Locking of adjustable ball joints}

Bone screws, systems for spinal correction and external fixators with ball joints allow a large degree of freedom during placement in or on the body. After the system has been applied the joint can be locked by heating a memory metal element. One part of the joint is made deformable by providing it with one or more slots. In these devices the recovery stress of the memory metal component is sufficient to prevent the movement of the joint at body temperature. After cooling the recovery stress drops to a very low value and

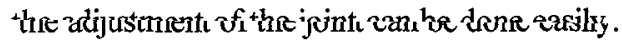


This effect can be reached in several ways, as shown in fig. 7:

a. the slotted ball is made of memory metal and fits in a rigid cup

b the ball and slotted cup are both made of conventional metal, but are locked by an external NiTi clamping element.

c the slotted steel ball has a central cavity that holds an expanding shape memory locking pin.

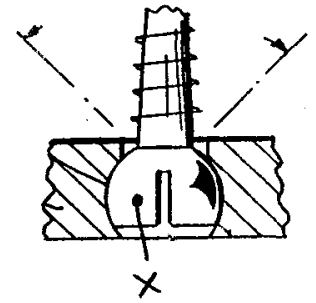

$7 \mathbf{a}$

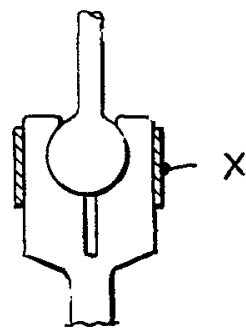

$7 \mathrm{~b}$

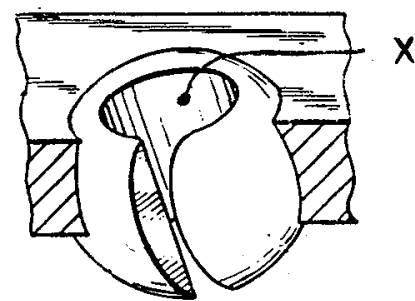

$7 \mathrm{c}$

Figure 7: Locking principles of a ball joint with a heat responsive NiTi-element as ball (a), external ring (b) or as internal pin ( not shown) in cylindrical cavity (c). The position of the NiTi is given with symbol X.

\section{SUPERELASTIC MEDICAL DEVICES}

Superelastic guide wires are used worldwide in all kinds of medical procedures. Sometimes these wires have a thin coating of PTFE or a hydrophilic layer like Slipskin ${ }^{\mathrm{TM}}$ [ 5 ] to reduce the friction in the catheter. Superelastic materials bring large advantages because of their excellent flexibility, kink resistance, pushability and torquability [6].

However, one disadvantage of superelasticity is that is is almost impossible for the surgeon to give a certain desired curvature to the tip if he wants to steer the guide wire into a certain direction. Of course this can be done by means of a heat treatment during production, but often this preset curve is not exactly what is needed.

A new type of guide wire that is under development now by $\mathrm{MMH}$ is superelastic, but by means of stretching the tip in its length direction a strain induced martensite is formed, that is stable at body temperature. This tip, once stretched, can now be bent without elastic recovery. The original straight annealed shape of the main part of the guide wire remains unchanged.

Other well known examples of superelastic devices are used in minimal invasive surgery, where a device is brought into the body in a delivery catheter or endoscope, while it is in a stress induced martensitic state. After the distal end of the instrument has reached the place where it has to be used, the memory metal part is pushed out of the delivery tube and the original preset shape is reached. Sometimes a part of the stem also has been heat treated to a preset curvature and when this part is also pushed out of the delivery tube, it is possible to work under an angle, with a steerable instrument.This principle is used in snares, baskets, grippers, steerable scissors, knives, suction tubes and optical systems [ 7,8,9].

The use of superelastic NiTiNb can bring additional advantages if products have to be more rigid, but still can be deformed elastically until $8 \%$ strain. The loading plateau of a NiTiNb-alloy, treated for optimum superelasticity, can be as high as $900 \mathrm{Mpa}$, while the unloading plateau is in the order of $500 \mathrm{Mpa}$. In fig. 8 the tensile curve is given for a regular binary NiTi-alloy and for a NiTiNb-alloy, both treated for superelastic behaviour.

During deployment of a constrained product the force is determined by the value of the unloading plateau, so a instrument made of NiTiNb is much stronger than a NiTi product of the same size. One aspect that has to be studied is the influence of the high stress level on the fatigue properties. 

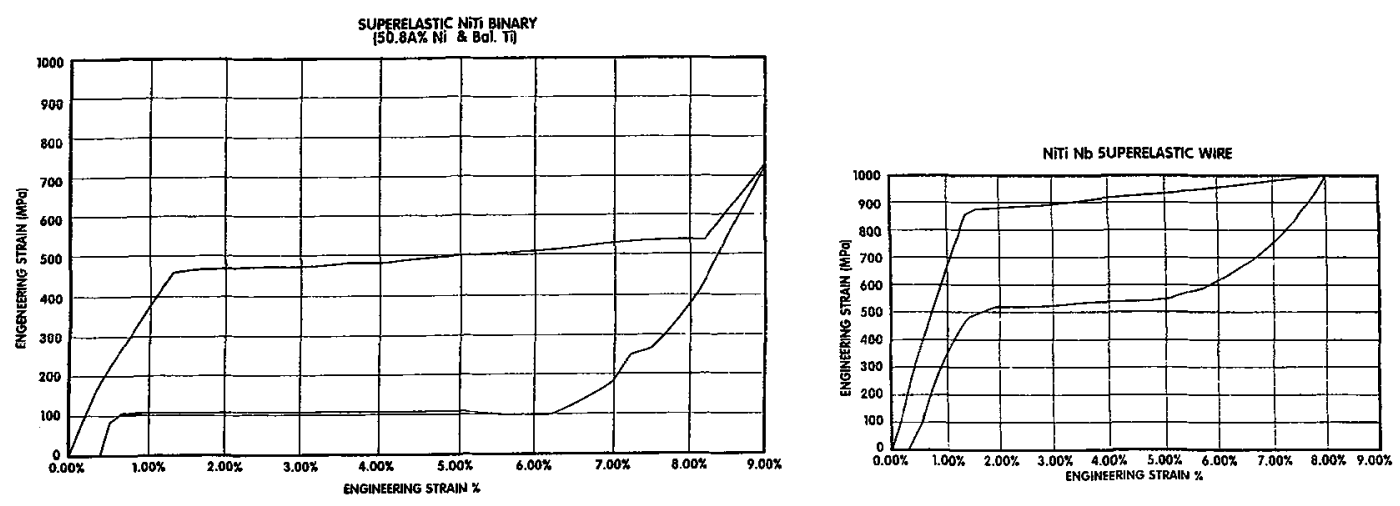

Figure 8: Comparison between the tensile curve for superelastic regular $\mathrm{NiTi}$ and $\mathrm{NiTiNb}$.

\subsection{Superelastic orthodontic devices}

Orthodontic archwires were the first biomedical mass application of memory alloys. While in the case of stainless steel wires the forces quickly relax during teeth movement concomitantly retarding this movement, NiTi-wires allow the teeth to move under almost constant force over a long treatment time and a much larger teeth displacement before the orthodontist has to tighten again.

There is a wide range of superelastic orthodontic arch wires for correction of the position of teeth and molars. Copper $\mathrm{NiTi}^{\mathrm{TM}}$, a tradename of Ormco, is a new type of wire, that has a transformation temperature just below body temperature and thus a very low plateau stress.

This makes the wire more sensitive to small temperature changes in the mouth which causes intermittent forces on the teeth. Another property of this Copper NiTi is the smaller stress hysteresis, compared to binary alloys. This means that there is less difference between the loading force ( during attachment) and the actual unloading (tooth-driving) force.

The T-gear is a neck strap for headgears with a superelastic tension spring. This device gives a fantastic freedom to the patient to move his head, while the reaction forces on the,molars hardly vary.

This device gives a large wearing comfort and excellent protection against overload.

Other examples are tension and compression springs to create space or close gaps between adjacent elements and heavy suture expanders.

\section{STENTS}

One of the most important types of applications today are NiTi-stents. They are made in a series of shapes, like braided wire, welded mesh, coil springs or slotted tube, dependent on the desired property or patent situation.

Memory metal stents are used as reinforcement of the wall of body cavities that are more or less collapsed by loss of elasticity or by external pressure.

In other cases they are used as grafting stents to hold an artery prosthesis in place to reinforce a weak part of an artery wall like an aneurisma. There are several types of stent deployment principles: 
a. Self expanding stents that deploy after after release from a delivery tube.

b. Temperature triggered stents with an As-temperature slightly above body temperature.

c. Balloon expandable, martensitic stents that can be removed after heating above Af.

An example is the use of the Memokath ${ }^{\mathrm{TM}}$ stent [ 10 ] to overcome the problem of urinary restrictions in male patients. The prostate can grow to such an extent that normal voiding is not possible anymore because the urethra is completely collapsed.

The stent has a transformation temperature sligthly above body temperature and it is put into place in a deformed, small diameter shape by means of a cystoscope.

After placement it is flushed during a few seconds with warm water of $45 \mathrm{C}$ which causes the recovery of the programmed shape.

The temperature hysteresis in the transformation will keep the device in its "warm" configuration and stability when the stent is cooled back to body temperature.

Removal is very easy by flushing with cold water of about $10 \mathrm{C}$. The stent becomes extremely soft then and it can be pulled out through a scope sheath as an almost straigth wire.

This possibility of using the temperature hysteresis is of special importance.

In contrary to conventional materials the memory metal enables the use of different structures at the same body temperature, dependent on the temperature path that has been followed.

In case of the stent it has either the "cold" soft mode or the "warm", stiffer and superelastic mode, both at $37 \mathrm{c}$.

The most used type of NiTi-stents is the self expanding, superelastic type. It brings the advantage of a gentle pressure against the artery wall, because during deployment the material works at its lower plateau stress. However, if by any external reason the artery might try to reclose, the stent will behave much stiffer, because it will then follow the upper plateau stress. The use of superelastic NiTiNb in stents may be interesting because of the larger stability and the possibility to reduce the wall thickness of a stent.

\section{SHAPEABLE MARTENSITIC STRUCTURES}

Martensitic structures can easily be bent into a range of shapes without the problems of excessive work hardening that occur in conventional metals.

In medical procedures it is sometimes difficult to reach specific places with a straight instrument and it can help if at least a part of this can be bent by the operator during surgery.

An endoscopic instrument that works with a steerable superelastic tip can be made with a bendable shape memory tube that acts as the delivery system for the precurved superelastic inner tube that holds the optical system in the tip [ 8 ]. In this way the surgeon has the possibility to operate in places in the body that could never be reached with conventional rigid endoscopes.

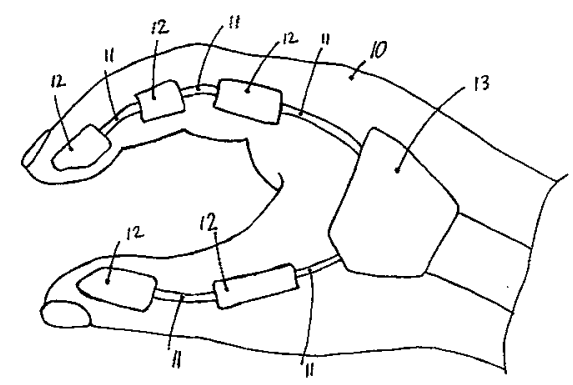

Figure 9: Principle of a cosmetic hand prosthesis with shapeable NiTi-elements (11) between rigid segments (12), embedded in a flexible silicon matrix (10). 
Another example, shown in fig. 9 , is a cosmetic hand prostheses, made of a silicon that surrounds a framework of martensitic NiTi-strips. The user can bend the fingers into a range of shapes by externally applied force and the fingers stay stable in their new position. On the places where the deformation should not occur, the strip is embedded in more rigid segments that have the same function as natural finger bones. The hands can be repositioned many thousands of times without failure of the strips [11 ].

\section{CONCLUSION}

Memory metals offer very interesting properties to designers of a wide range of products. If possible, the design has to be made in such a way that a minimum amount of material is used to achieve a better performance, higher reaction speed and a smaller size. Sometimes the forces that are generated by the memory metal, can be amplified by simple mechanical solutions.

The producers of memory metals are focussing on the market for medical applications.

Although until now in most cases only one of the specific properties of shape memory alloys is used per application, it may be interesting to develop a new generation of implants and instruments that uses combinations of these properties. An example can be a steerable instrument for minimal invasive surgery with a distal shape memory locking element for rapid change of tools. The instrument can be made modular to enable customization in the operation room. A part of the delivery tube can be of a shapeable, martensic material, while the inner part is superelastic, with a preset curvature to get the steerability.

\section{References}

[1] IPS. Patent EP 0461075 B1 "Heat responsive memory metal actuator."

[2] P.A.Besselink, "Procedure for the calculation of the geometry of a resistance heated NiTi-actuator", proc. Actuator 1996, Bremen.

[3] P.A.Besselink and R.C.L.Sachdeva, "Applications of shape memory effects",J. de Physique IV, Colloque C8, volume 5, decembre 1995, pp. 111-116.

[4] J. Hesselbach and M.Kristen, "Shape memory actuators as electrically controlled positioning elements", proc. Actuator 1992, Bremen, pp 85-91.

[5] "Coated wire for medical applications", Company brochure, Belden Wire \& Cable B.V. Venlo, The Netherlands.

[6] R.Fernald, D.Fritz, C.Sievert and J.Stice, "NiTi:The material of choice for high performance guidewires", Proc. SMST-94, Asilomar March 7-10, (1994) pp. 341-345.

[7] S.S.Moran, "Flexible Instruments in Minimal Invasive Surgery", proceedings SMST, ASILOMAR March 7-10, (1994) pp. 411-416.

[8] P.P.Poncet and R.Zadno, "Applications of superelastic Ni-Ti in laparoscopy", proc. SMST, Asilomar March 7-10, (1994) pp. 421-426.

[9] R.C.L.Sachdeva and P.A.Besselink, "Instrument for endoscopic-type procedures", US-patent $5,607,435$.

[10] B.M.Soni, S.Vaidyanatham, K.R.Krishnan, “ Use of Memokath, a second generation urethral stent for relief of urinary retention in male spinal cord injured patients" Paraplegia 32 (1994), 480-488.

[11] Patent PCT/NL96/00182 "Transformable structure". 